\title{
Fucoidan-New Principle Prevention and Treatment of Diabetes
}

\author{
Emil Mukhamejanov ${ }^{1}$, Galina Kon ${ }^{1}$, Sara Erjanova ${ }^{2}$, Aray Kirgizbaeva ${ }^{2}$ and Elizabeth Muhamadieva ${ }^{2}$ \\ 1. JSC "Scientific Center for Anti-Infectious Drugs" \\ 2. Asfendiyarov Kazakh National Medical University
}

\begin{abstract}
Most of patients with diabetes mellitus are not able to adhere to the basic principles of maintaining glucose homeostasis by decrease consumption of carbohydrates from food and increasing their utilization for physical activity. Pharmacological correction developing disorders are not always effective and have side effects. That is why necessary search for nutraceuticals with high bio-active properties does not have side effects. This review being considered possibility using fucoidan is sulfated polysaccharide of brown algae to maintain glucose homeostasis by reducing the rate absorption of glucose from the intestine and increasing its utilization by muscle fibers as a nutraceutical for patients with diabetes mellitus. In this plan, fucoidan should be considered as a functional food product for the prevention and treatment of diabetes mellitus.
\end{abstract}

Key words: Diabetes, fucoidan, prevention, treatment.

\section{Introduction}

Basic principle prevention and treatment of diabetes mellitus is decreased using carbohydrates (diet) and increasing its utilization (physical activity). Indeed, people who adhere to these rules, will control the level of glycaemia. Unfortunately, a person is a weak creature and he wants to eat and lie down on the sofa, so most of the population does not follow the basic principles of a healthy lifestyle (HLS) and the number of people with diabetes increases [1]. Everyone wants to get a "miracle pill" that would allow them to live a full life even if they do not follow the principles of HLS. Today people creat a lot of technology and medical substances for decrease and utilization carbohydrates and increase their absorption by peripheral tissues, but in most cases these drugs have serious side effects, therefore, in recent years, particular attention has been paid to the search for food compounds with high bioactivity or nutraceuticals (derivatives from word nutrition and pharmaceutics).

Corresponding author: Emil Mukhamejanov, DMS, professor, research field: biochemical nutrition.
Special interest in this plan is representing sea products (of plant and animal origin). Life originated from the sea and in the seaflooding all bioactive compounds of land. Definitely, epidemiological evidence suggests that people in Japan and South Korea who eat seafood in large quantities have a high longevity and good health. Their diet contains a large amount of algae [2], they are claimed to be a good supply of key nutrients including carbohydrates, protein and minerals [3], as well as a rich source of health-promoting compounds capable of acting on a wide spectrum of disorders and/or diseases [4]. Note also that the Japanese have the world's longest life expectancy, which has been partly associated with their different dietary patterns, and of course, the regular consumption of macroalgae $[5,6]$ the most important bio active component of which is the sulfated polysaccharide fucoidan.

In human nutrition are usually present two polysaccharides: starch or glucose monosaccharide polymer and inulin or fructose monosaccharide polymer. In fucoidan, $80 \%$ of the monosaccharides fall on fucose monosaccharide, which by chemical 
structure is galactose without a hydroxyl group in the sixth position and is in the L-configuration, while all monosaccharides are in the D-configuration [7]. In the past two decades there have been many publications on fucoidan due to its wide bio-activity.

At the present time shown: antioxidant [8], antibacterial [9], antiviral [10], anticoagulant [11], anti-inflammatory [12], antitumor [13] properties of fucoidan and it is recommended to use fucoidan as a product of functional nutrition for different diseases. Such a wide range of biological effects of fucoidan causes mistrust, therefore legitimate to ask a myth or reality [14].

In this review, we will focus on the antidiabetic properties of fucoidan. It should be noted about the effect of fucoidan in diabetes have been carried out on extensive preclinical studies on invitro models (on separate cell systems) and in vivo (on various laboratory animals). The first phase of clinical trials - tolerability and safety [15] has been completed, as well as pilot studies were conducted on the second phase of clinical trials in observations on patients with diabetes.

The inhibition of $\alpha$-glucosidase is an effective therapeutic approach for type 2 diabetes mellitus that involves decreasing postprandial hyperglycemia. In study the $\alpha$-glucosidase and $\alpha$-amylase inhibitory effects of 11 fucoidans extracted from different brown seaweeds were evaluated [16]. Although no significant $\alpha$-amylase inhibition was observed, fucoidan from Fucusvesiculosus (FvF) showed the highest $\alpha$-glucosidase inhibitory activity, with an $\mathrm{IC}_{50}$ (drug concentration causing inhibition of the enzyme on 50\%) value of $67.9 \mu \mathrm{g} / \mathrm{mL}$. In addition, $\mathrm{FvF}$ at a concentration of $200 \mu \mathrm{g} / \mathrm{mL}$ displayed very mild cytotoxicity to IEC-6 cells as indicated by the MTT assay. An in vivo study indicated that FvF decreased the fasting blood glucose and hemoglobin A1c (HbA1c) levels of $\mathrm{db} / \mathrm{db}$ mice, with minimal effect on their weight. Therefore, our present in vitro and in vivo studies demonstrated that FvF could be a promising $\alpha$-glucosidase inhibitor for the treatment of type 2 diabetes mellitus. Fucoidan in concentrations of 62.5, 125 and $250 \mu$ ginhibits amylase by 25, 63 and 99\%, respectively [17]. The $\mathrm{IC}_{50}$ value was $103.83 \mu \mathrm{g}$, which is lower than for acarbose (16mg), a pharmacological drug used in the treatment of diabetes. High inhibitory activity ( $\mathrm{IC}_{50}$ was $133 \mu \mathrm{g}$ ) was also shown for glucosidase, which is an order of magnitude lower than for acarbose [18].

Similar results were obtained for fucoidan from $\mathrm{F}$. vesiculosus, when the $\mathrm{IC}_{50}$ was $0.049 \mathrm{mg} / \mathrm{mL}$ [19]. The differences in the $\mathrm{IC}_{50}$ are due to the use of various fucoidan preparations or methods for its isolation from various types of brown algae.

An important cause of diabetes is the impaired $\beta$-cell activity of the pancreas, therefore preventing their death is an important aspect of preventing diabetes. Fucoidan has helped increase glucagon-likepeptide-1 receptor (GLP-1R) andsirtuin 1 (Sirt-1) duo toactivation AMPK/GAPDH/PDX-1 cascade in STZ-treated $\beta$ cells of mice, which lead to decreasing their apoptosis and preventing dysfunction of $\beta$-cells [20]. Fucoidan in doses 200 or 1,200 $\mathrm{mg} / \mathrm{kg} /$ bodyweight/day contributed to decreasing level of glucose in blood on $22 \%$ and $34 \%$, in aloxane rats, respectively, i.e. is noted a dose-dependent effect [21].

Under the influence of fucoidan, there was an increase level of insulin in serum, lower cholesterol, triglycerides and LDL. Apparently, fucoidan due to the ability to reduce amount of apoptosis of $\beta$-cells contributes to the improvement of insulin secretion, which leads to its hypoglycemic effect. Really, fucoidanat concentration of $75 \mathrm{mg} / \mathrm{kg}$ body weight for 13 weeks contributed to increasing insulin secretion in Male Goto-Kakizaki (GK) mice indose-dependent manner [22], while its effect was much higher than for the pharmacological preparation glybenclamide.

Type 2 diabetes (2D) is a multi-systemic disease which characterized by impaired activity of various organs and systems. Control postprandial (after a meal) hyperglycemia an important factor in the prevention 
and treatment of 2D. In linear mice with diabetes was studied influence fucoidan glucose tolerance test, food intake, weight, fasting glucose and biochemical parameters in $\mathrm{db} / \mathrm{db}$ mice. The using of fucoidan indose $200 \mathrm{mg} / \mathrm{kg}$ is prevented hyperglycemia in control and diabetic mice [23].

Chemical structures of drugs fucoidan depend on the type of algae, their place of growth, methods of isolation, weather conditions and other factors [24], so that it cannot be marketed as a drug, that requires the clarity of the chemical structure, and is equal to the product of the power of the functional type of action.

Decrease glycaemia to laboratory animals noted in type 1 diabetes [25]. Low molecular weight fucoidan (LMWF) in dose $100 \mathrm{mg} / \mathrm{kg} /$ day-treated group showed robust improvements on STZ-induced body weight-loss, hypertension, and hyperlipidaemia as indicated by decreased serum level of total cholesterol, triglyceride, and low density lipoprotein cholesterol; while probucol, a lipid-modifying drug with antioxidant properties, displayed mild effects. In addition, LMWF appreciably ameliorated STZ-elicited hyper-responsiveness and oxidative stress in aortic smooth muscles as indicated by decreased superoxide level, increased glutathione content and higher superoxide dismutase activity. Increase level of free radicals (FR) and development manifestations of oxidative stress is important pathogenic part of the development of negative manifestations in diabetes mellitus, with which the development of disorders of various organs and body systems. Of course, organs with low antioxidant protection are primarily oxidative damaged. Historical facts indicate that the Spanish conquistadors at highland conditions due to the increased level of FR noted a violation of the activity of the ovaries and the development of infertility, which prevented the intake of the antioxidant vitamin $\mathrm{E}$, therefore it was mistakenly attributed to the reproduction vitamin. Under the influence of streptozotocin in rats caused diabetes. Intake for 15 days, $50 \mathrm{mg} / \mathrm{kg}$ of fucoidan minimized the negative effect of diabetes on the violation of the testicles [26], which the authors attributed to the antioxidant effect of fucoidan.

In patients with diabetes, the risk of damage to peripheral arterial disease (PAD) and the development of tissue ischemia increases, which can lead to a decrease energy supply of the tissues (especially the lower limbs), which are manifested in the symptom “diabetic foot”. Type 2 diabetic Goto-Kakizaki (GK) rats were made $\mathrm{PAD}$ by injection of sodium laurate into femoral artery. LMWF (20, 40 or $80 \mathrm{mg} / \mathrm{kg} /$ day) or cilostazol (100 mg/kg/day) were given to diabetic PAD rats for 4 weeks, respectively [27]. They revealed that LMWF markedly ameliorated foot ulceration and claudication, and improved the plantar perfusion by reversing hyper reactive platelet aggregation, ameliorating endothelium-dependent vasodilation and revascularization on diabetic PAD rats.

The violation activity pancreas is an important reason for the development of 2D. On rats Goto-Kakizaki (controlrate Wistar) effect of fucoidan on insulin secretion was evaluated [28]. Oral intake fucoidan for 13 weeks contributed to a decrease in sugar and insulin, as well as oral fucoidan administration for 13 weeks contributed to a decrease in sugar and insulin, as well as of cyclic adenosine monophosphate (cAMP).

Fucoidan having antioxidant properties prevents the development of hypertension (increase in aortic tone) and hyperlipidemia [29]. Indeed, diabetic patients have a high risk of endothelial dysfunction and vascular damage due to hyperglycemia, lipedema and oxidative stress. Atherosclerosis, hypertension and peripheral vascular disorders are characteristic of these patients. Endothelial nitrite oxidase (NO) regulates vascular homeostasis through vasodilation and the protection effects of damaging agents. A decrease in NO leads to an increase in pro-inflammatory factors, in particular, nuclear factors and further increases endothelial inflammation and atherosclerosis, therefore, the development of endothelial dysfunction contributes to 
the imbalance of NO in the early stages of diabetes. Violation activity of endothelial NO synthase (eNOS) is an important pathogenetic factor for endothelial dysfunction in diabetes; therefore, improving NO functioning is a target for endothelium and reducing cardiovascular system (CVS) disorders in diabetes. The effect of fucoidan on vascular disorders was assessed in rats aged 13-14 weeks with high hyperglycemia. It is noted that decrease manifestations of disorders in rats with diabetes, which only slightly decreased with the use of probucol (the usual pharmacologic drug).

Great importance in mechanism development of diabetes is violation of lipid metabolism, when lipids act as a competitor for the process formation of ATP and thereby inhibit glucose uptake (Randle cycle). In this plane have big interest presents a study [30] which conducted on L6 myotubes of mice with 2D. Under effects of metmorphine and fucoidan, there was an improvement in glucose utilization by muscle fibers (in terms of 2-deoxyglucose utilization) and a decrease in triglyceride and cholesterol levels. At the same time, the effect of fucoidan is comparable to the effect of metmorphine (the gold standard in the treatment of diabetes), which indicates the high potential of using fucoidan as a product for functional nutrition. According to the authors, the effect of fucoidan and metmorphine occurs indirectly through their effect on AMP activated protein kinase (AMPK), because giving an inhibitor of this kinase removed the effect of fucoidan and metmorphine on the carbohydrate and lipid metabolism.

As mentioned above, hyperglycemia is an important pathogenetic link for the development of disorders in diabetes. At hyperglycemia activates the process glycation of proteins, in particular hemoglobin, therefore, the determination of glycated hemoglobin is a marker degree development of pathological manifestations in diabetes. In this regard, it is interesting to know how fucoidan affects the glycation process. Fucoidan reduces the reaction of non-enzymatic glycation (Maillard reaction) of collagen in the presence of glucose [31]. This indicates that under the influence of fucoidan, the amount of protein glycation decreases, i.e. use of fucoidan is a targeted way to prevent and treat the negative effects of hyperglycemia.

Diabetic retinopathy is a fairly common complication of diabetes. Hyperglycemia promotes oxidative damage to retina cells and the development of pathological changes. Excessive production of FR causes the development of cell dysfunction, which causes hyperglycemic damage in diabetes. An cell culture ARPE-19 is shown, that glycemia contributes to the generation of $\mathrm{FR}$, which lead to structural violation of cell [32]. Adding fucoidan had a protective effect on ARPE-19 cells on the background of glycaemia. The mechanism of the negative influence of hyperglycemia is the release of calcium, and fucoidan reduced the release of calcium during hyperglycemia.

Increased FR levels and the development of oxidative stress underlie pathological manifestations in diabetes mellitus. On 25 fat volunteers were conducted randomized study effects of daily fucoidan with double placebo control [33]. Thirteen individuals who intake $500 \mathrm{mg}$ of fucoidan daily before breakfast and 12 received a placebo for 3 months. All were evaluated glucose, cholesterol, cholesterol of HDL, cholesterol of LDL (LDL-C), triglycerides and insulin, and glucose homeostasis was assessed by (HOMA). The results showed a significant decrease in diastolic pressure (71.7 \pm 12.2 vs. $67.8 \pm 13.8 \mathrm{mmHg} ; P<0.05)$ and LDL-C (3.1 \pm 0.5 vs. $2.7 \pm 0.6 \mathrm{mmol} / \mathrm{l} ; P<0.01)$ with increasing insulin levels $(60.6 \pm 24.0$ vs. $78.6 \pm$ 32.4 pmol/l; $P<0.05)$, HOMA $\beta$-cell (35.0 \pm 20.8 vs. $50.6 \pm 18.7 ; P<0.05)$ when giving fucoidan. It was concluded that giving fucoidan for 3 monthly individuals decrease blood pressure and LDL-C, increasing insulin secretion. Although the authors write that they were observing for obese individuals, but obese individuals usually have type 2 diabetes.

Thus, these data indicate a high potential for using 
fucoidan for the prevention and treatment of diabetes patients.

Lipids are an important pathogenetic factor in the development of diabetes; therefore, improving the lipid spectrum is the principle of prevention and treatment of diabetes. Adipose tissue, which is primarily composed of adipocytes, is crucial for maintaining energy and metabolic homeostasis. Adipogenesis is thought to occur in two stages: commitment of mesenchymal stem cells to a preadipocyte fate and terminal differentiation. Cell shape and extracellular matrix remodelling have recently been found to regulate preadipocyte commitment and competency by modulating WNT and RHO-family GTPase signalling cascades. Adipogenic stimuli induce terminal differentiation in committed preadipocytes through the epigenomic activation of peroxisome proliferator-activated receptor- $\gamma(\operatorname{PPAR} \gamma)$ [34]. The coordination of PPAR $\gamma$ with CCAAT/enhancer-binding protein (C/EBP) transcription factors maintains adipocyte gene expression. Improving our understanding of these mechanisms may allow us to identify therapeutic targets against metabolic diseases that are rapidly becoming epidemic globally.

Fucoidan treatment inhibits differentiation 3T3-L1 preadipocytes, evidenced by decreased lipid accumulation and down regulation of adipocyte markers. Fucoidan then inhibited the expression of both early CCAAT-enhancer-binding proteins alpha (C/EBPalpha) and peroxisome proliferator-activated receptors gamma (PPARgamma) and late activating protein 2 (aP2) adipogenic transcription factors, which is a crucial role for adipocyte development. Moreover, fucoidan inhibited the early activation of p38 MAPKs, extracellular signal-regulated kinases (ERK) and Jun $\mathrm{N}$-terminal kinase (JNK) [35]. Overall, these findings are a strong indication that fucoidan might inhibit adipogenesis in 3T3-L1 preadipocytes, due to inhibition of the MAPK signaling pathway that involves adipogenic transcription factors.

\section{Conclusions}

The sulfated polysaccharide fucoidan promotes maintaining homeostasis of glucose due to the decrease rate of its absorption in the gut and increase in utilization of muscle fibers, which leads to the prevention of the development of glycaemia and lipedema. The positive effect fucoidan at diabetes mellitus linked to its antioxidant properties and ability to reduce manifestations of apoptosis, in particular, beta cells of pancreas, which save the ability of cells to secrete insulin. Thus, the sulfated polysaccharide fucoidan should be attributed to functional foods and should be used in diet therapy for the prevention and treatment of diabetes mellitus.

\section{References}

[1] Wild, S., Roglic, G., Green, A., Sicree, R., and King, H. 2004. "Global Prevalence of Diabetes: Estimates for the Year 2000 and Projections for 2030.” Diabetes Care 27: 1047-53.

[2] Iso, H., and Kubota, Y. 2007. "Nutrition and Disease in the Japan Collaborative Cohort Study for Evaluation of Cancer (JACC).” Asian Pac. J. Cancer Prev. 8: 35-80.

[3] Rajapakse, N., and Kim, S. K. 2011. "Nutritional and Digestive Health Benefits of Seaweed.” Adv Food Nutr Res. 64: 17-28.

[4] Bocanegra, A., Bastida, S., Benedi, J., Rodenas, S., and Sanchez-Muniz, F. J. 2013. "Characteristics and Nutritional and Cardiovascular-Health Properties of Seaweeds.” J. Med. Food 12: 236-58.

[5] Cardoso, S., Carvalho, L., Silva, P., Rodrigues, M., Pereira, O., and Pereira, L. 2014. "Bioproducts from Seaweeds: A Review with Special Focus on the Iberian Peninsula.” Curr. Org. Chem. 18: 896-917.

[6] Cardoso, S., Pereira, O., Seca, A., Pinto, D., and Silva, A. 2015. "Seaweeds as Preventive Agents for Cardiovascular Diseases: From Nutrients to Functional Foods.” Mar. Drugs 13: 6838-65.

[7] Li, B., Lu, F., Wei, X., and Zhao, R. 2008. "Review Fucoidan: Structure and Bioactivity.” Molecules 13: 1671-95.

[8] de Souza, M. C. R., Marques, C. T., Dore, C. M. G., da Silva, F. R. F., Rocha, H. A. O., and Leite, E. L. 2007. "Antioxidant Activities of Sulfated Polysaccharides from Brown and Red Seaweeds.” J.Appl.Phycol. 19: 153-60. 
[9] Souza, M. C., Penido, C., Costa, M. F. S., and Henriques M. G. 2008. "Mechanisms of T-Lymphocyte Accumulation during Experimental Pleural Infection Induced by Mycobacterium bovis.” BCG'Infect Immun. 76: 5686-93.

[10] Negishi, H., Mori, M., Mori, H., and Yamori, Y. 2013. "Supplementation of Elderly Japanese Men and Women with Fucoidan from Seaweed Increases Immune Responses to Seasonal Influenza Vaccination.” J Nutr. 143: 1794-8.

[11] Pereira, M. S., Mulloy, B., and Mourão, P. A. 1999. "Structure and Anticoagulant Activity of Sulfated Fucans. Comparison between the Regular, Repetitive, and Linear Fucans from Echinoderms with the More Heterogeneous and Branched Polymers from Brown Algae.” J. Biol. Chem. 274: 7656-67.

[12] Kim, K. J., and Lee, B. Y. 2012. "Fucoidanfrom the Sporophyll of Undariapinnatifida Suppresses Adipocyte Differentiation by Inhibition of Inflammation-related Cytokines in 3T3-L1 Cells.” Nutr Res. 32: 439-47.

[13] Huang, T-H., Chiu, Y-H., Chan, Y-L., Chiu, Y-H., Wang, H., Huang, K-C., Li, T-L., Hsu, K-H., and Wu, C-J. 2015. "Prophylactic Administration of Fucoidan Represses Cancer Metastasis by Inhibiting Vascular Endothelial Growth Factor (VEGF) and Matrix Metalloproteinases (MMPs) in Lewis Tumor-Bearing Mice.” Mar. Drugs 13: 1882-900.

[14] Ermakova, S., Kusaykin, M., Trincone, A., and Zvyagintseva, T. 2015. "Are Multifunctional Marine Polysaccharides a Myth or Reality?” Front Chem. 3: 39.

[15] Abe, S., Hiramatsu, K., Ichikawa, O., Kawamoto, H., Kasagi, T., Miki, Y., Kimura, T., and Ikeda, T. 2013. "Safety Evaluation of Excessive Ingestion of Mozuku Fucoidan in Human.” J.Food Sci. 78: 648-51.

[16] Shan, X., Liu, X., Hao, J., Cai, C., Fan, F., Dun, Y., Zhao, X., Liu X., Li, C., and Yu, G. 2016. "In Vitro and in Vivo Hypoglycemic Effects of Brown Algal Fucoidans.” Int.J.Biol.Macromol. 82: 249-55.

[17] Lakshmana, S. S., Vinoth, K. T., Geetharamani, D., Suja, G., Yesudas, R., and Chacko, A. 2015. "Fucoidan-An $\alpha$-amylase Inhibitor from Sargassumwightii with Relevance to NIDDM.” Int.J.Biol.Macromol. 81: 644-7.

[18] Vinoth, K. T., Lakshmanasenthil, S., Geetharamani, D., Marudhupandi, T., Suja, G., and Suganya, P. 2015. "Fucoidan-A $\alpha$-D-glucosidase Inhibitor from Sargassumwightii with Relevance to Type 2 Diabetes Mellitus Therapy.” Int.J.Biol.Macromol. 72: 1044-7.

[19] Kim, K. T., Rioux, L. E., and Turgeon, S. L. 2014. "Alpha-amylase and Alpha-glucosidase Inhibition is Differentially Modulated by Fucoidan Obtained from Fucus vesiculosus and Ascophyllum nodosum." Phytochemistry 98: 27-33.
[20] Yu, W. C., Chen, Y. L., Hwang, P. A., Chen, T. H., and Chou, T. C. 2017. "Fucoidan Ameliorates Pancreatic $\beta$-cell Death and Impaired Insulin Synthesis in Streptozotocin-treated $\beta$ Cells and Mice via a Sirt-1-dependent Manner.” Mol. Nutr. Food Res. 61 (10).

[21] Wang, J., Jin, W., Zhang, W., Hou, Y., Zhang, H., and Zhang, Q. 2013. "Hypoglycemic Property of Acidic Polysaccharide Extracted from Saccharina Japonica and Its Potential Mechanism.” Carbohydr. Polym. 95: 143-7.

[22] Jiang, X., Yu, J., Ma, Z., Zhang, H., and Xie, F. 2015. "Effects of Fucoidan on Insulin Stimulation and Pancreatic Protection via the cAMP Signaling Pathway in Vivo and in Vitro.” Mol.Med.Rep. 12: 4501-7.

[23] Kim, K. J., Yoon, K. Y., and Lee, B. Y. 2012. "Fucoidan Regulate Blood Glucose Homeostasis in C57BL/KSJ $\mathrm{m}+/+\mathrm{db}$ and C57BL/KSJ db/db Mice.” Fitoterapia 83: 1105-9.

[24] Li, B., Lu, F., Wei, X., and Zhao, R. 2008. "Fucoidan: Structure and Bioactivity.” Molecules 13: 1671-95.

[25] Liang, Z., Zheng, Y., Wang, J., Zhang, Q., Ren, S., Liu, T., Wang, Z., and Luo, D. 2016. "Low Molecular Weight Fucoidan Ameliorates Streptozotocin-induced Hyper-responsiveness of Aortic Smooth Muscles in Type 1 Diabetes Rats.” J. Ethnopharmacol. 191: 341-9.

[26] Ersoy, O., and Kizilay, G. 2018. "Effects of Fucoidan on Diabetic Rat Testicular Tissue.” Biotech. Histochem. 93: 277-85.

[27] Wang, Z., Liu, T., Chen, X., You, H., Zhang, Q., Xue, J., Zheng, Y., and Luo, D. 2018. "Low Molecular Weight Fucoidan Ameliorates Hindlimb Ischemic Injury in Type 2 Diabetic Rats.” J Ethnopharmacol. 210: 434-42.

[28] Jiang, X., Yu, J., Ma, Z., Zhang, H., and Xie, F. 2015. "Effects of Fucoidan on Insulin Stimulation and Pancreatic Protection via the cAMP Signaling Pathway in Vivo and in Vitro.” Mol Med Rep. 12: 4501-7.

[29] Cui, W., Zheng, Y., Zhang, Q., Wang, J., Wang, L., Yang, W., Guo, C., Gao, W., Wang, X., and Luo, D. 2014. “Angiogenesis, Cardiovascular and Pulmonary Systems Low-molecular-weight Fucoidan Protects Endothelial Function and Ameliorates Basal Hypertension in Diabetic Goto-Kakizakirats Laboratory.” Investigation 94: 382-93.

[30] Jeong, Y-T., Kim, Y. D., Jung, Y-M., Park, D-C., Lee, D-S., Ku, S-K., Li, X., Lu, Y., Chao, G. H., Kim, K-J., Lee, J-Y., Baek, M-C., Kang, W., Hwang, S-L., and Chang, H. W. 2013. "Low Molecular Weight Fucoidan Improves Endoplasmic Reticulum Stress-Reduced Insulin Sensitivity through AMP-Activated Protein Kinase Activation in L6 Myotubes and Restores Lipid Homeostasis in a Mouse Model of Type 2 Diabetes.” Molecular Pharmacology 84: 147-57.

[31] Pielesz, A., and Paluch, J. 2014. "Fucoidan as an Inhibitor of Thermally Induced Collagen Glycation Examined by 
Acetate Electrophoresis.” Electrophores 35: 2237-44.

[32] Li, X., Zhao, H., Wang, Q., Liang, H., and Jiang, X. 2015. "Fucoidan Protects ARPE-19 Cells from Oxidative Stress via Normalization of Reactive Oxygen Species Generation through the Ca2+-dependent ERK Signaling Pathway.” Mol.Med.Rep. 11: 3746-52.

[33] Hernández-Corona, D. M., Martínez-Abundis, E., and González-Ortiz, M. 2014. "Effect of Fucoidan Administration on Insulin Secretion and Insulin
Resistance in Overweight or Obese Adults.” JMedFood 17: 830-42.

[34] Cristancho, A. G., and Lazar, M. A. 2011. "Forming Functional Fat: A Growing Understanding of Adipocyte Differentiation.” Nat Rev Mol Cell Biol. 12: 722-34.

[35] Kim, K. J. Lee, O. H., and Lee, B. Y. 2010. "Fucoidan, a Sulfated Polysaccharide, Inhibits Adipogenesis through the Mitogen-activated Protein Kinase Pathway in 3T3-L1 Preadipocytes.” Life Sci. 86: 791-7. 